\title{
Quantification of right ventricular volume in dogs: a comparative study between three-dimensional echocardiography and computed tomography with the reference method magnetic resonance imaging
}

Anne K Sieslack ${ }^{\dagger}$, Peter Dziallas ${ }^{\dagger}$, Ingo Nolte ${ }^{\dagger}$, Patrick Wefstaedt ${ }^{\dagger}$ and Stephan O Hungerbühler ${ }^{*}$

\begin{abstract}
Background: Right ventricular (RV) volume and function are important diagnostic and prognostic factors in dogs with primary or secondary right-sided heart failure. The complex shape of the right ventricle and its retrosternal position make the quantification of its volume difficult. For that reason, only few studies exist, which deal with the determination of RV volume parameters. In human medicine cardiac magnetic resonance imaging (CMRI) is considered to be the reference technique for RV volumetric measurement (Nat Rev Cardiol 7(10):551-563, 2010), but cardiac computed tomography (CCT) and three-dimensional echocardiography (3DE) are other non-invasive methods feasible for RV volume quantification. The purpose of this study was the comparison of 3DE and CCT with CMRI, the gold standard for RV volumetric quantification.

Results: 3DE showed significant lower and CCT significant higher right ventricular volumes than CMRI. Both techniques showed very good correlations $(R>0.8)$ with $C M R I$ for the volumetric parameters end-diastolic volume (EDV) and end-systolic volume (ESV). Ejection fraction (EF) and stroke volume (SV) were not different when considering CCT and CMRI, whereas 3DE showed a significant higher EF and lower SV than CMRI. The 3DE values showed excellent intra-observer variability $(<3 \%)$ and still acceptable inter-observer variability $(<13 \%)$.

Conclusion: $\mathrm{CCT}$ provides an accurate image quality of the right ventricle with comparable results to the reference method CMRI. CCT overestimates the RV volumes; therefore, it is not an interchangeable method, having the disadvantage as well of needing general anaesthesia. 3DE underestimated the RV-Volumes, which could be explained by the worse image resolution. The excellent correlation between the methods indicates a close relationship between 3DE and CMRI although not directly comparable. 3DE is a promising technique for RV volumetric quantification, but further studies in awake dogs and dogs with heart disease are necessary to evaluate its usefulness in veterinary cardiology.
\end{abstract}

Keywords: Right ventricular volume, Dog, Three-dimensional echocardiography, Magnetic resonance imaging, Computed tomography

\footnotetext{
* Correspondence: Stephan.Hungerbuehler@tiho-hannover.de

${ }^{\dagger}$ Equal contributors

Small Animal Clinic, University of Veterinary Medicine Hannover, Foundation, Bünteweg 9, Hannover D-30559, Germany
} 


\section{Background}

The quantification of right ventricular (RV) volume and function is of major clinical relevance regarding morbidity and mortality in patients with pulmonary hypertension, congenital heart disease and congestive heart failure [1-5]. The complex anatomical structures of the right ventricle, such as the crescent shape, the thin wall wrapped around the left ventricle, and the trabeculae of the right ventricular wall, make the evaluation of $\mathrm{RV}$ function challenging [6,7]. Consequently, a twodimensional depiction is not adequate for accurate RV assessment because the right ventricle can only be incompletely visualised in a single view $[2,8]$. Despite this inaccuracy, two-dimensional echocardiography is the most widely used technique for investigations of the right ventricle in human medicine [5].

The use of three-dimensional (3D) methods promises more accuracy by showing the right ventricle in many planes, enabling a calculation of RV volume independently from model assumptions of ventricular geometry. In many human studies, RV volumetric quantification showed good correlations between $3 \mathrm{DE}$ and cardiac magnetic resonance imaging (CMRI) with lower volumes using 3DE $[6,9,10]$. However, an in vitro study with excised porcine hearts showed good agreement between the 3DE Simpson method and RV volume determination by means of a silicon latex cast [11]. Likewise an in vivo study of 5 dogs, which compared intracavitary balloon measurements and $3 \mathrm{DE}$ values, was able to show an accurate assessment of RV volume by means of 3DE [12]. The major advantage of $3 \mathrm{DE}$ in veterinary medicine is the unnecessity for general anaesthesia, especially in patients with heart disease. The sectional imaging techniques CMRI and cardiac computed tomography (CCT) provide 3D information of the heart with high spatial resolution $[13,14]$. Both techniques have proven to be reliable imaging modalities for depicting morphological and anatomical heart structures under physiological and pathological conditions in dogs [15-17]. The need for anaesthesia is the main reason for their reduced use in veterinary medicine. In human medicine, CMRI is regarded as the gold standard for RV volume and function assessment [18] because of its high temporal and spatial resolution and its detailed soft tissue contrast, resulting in high accuracy and reproducibility of the measurements. For this reason, CMRI is considered to be the reference method in many studies investigating RV function $[9,10,19,20]$. In veterinary medicine, the use of CMRI for RV determination is rare. Only a few studies have been concerned with the right ventricle. In one study it was concluded that technical limitations hamper a good image quality of the moving heart in dogs [21]. However, a more recent study, determining the RV mass in dogs, found accurate results for in vivo CMRI values in comparison to ex vivo measurements [22].
As already mentioned, CCT can be used for cardiac visualisation as well [16]. Nevertheless, in veterinary medicine its use for RV assessment has not been established yet. Considering that veterinary clinics are equipped with a CT scanner more often than an MRI scanner, the evaluation of $\mathrm{RV}$ volumetric measurement with CCT is of importance. Therefore, this study focuses on the comparison of RV volumetric quantification by means of $3 \mathrm{DE}$ and CCT in comparison with the gold standard CMRI.

\section{Methods}

The study was approved by the Ethical Committee of the Lower Saxony State Office for Consumer Protection and Food Safety (33.9-42502-05-11A133). All study participants were clinic-owned, healthy beagles from the Small Animal Clinic of the University of Veterinary Medicine Hannover, Foundation. The dogs (7 male, 3 female) had a mean age of 6.5 years $( \pm 3.26$ standard deviation [SD]) and an average body weight of $16.6 \mathrm{~kg}$ $( \pm 2.08 \mathrm{SD})$. All dogs included in the study had on the day before the study undergone normal parameters of the physical examination, blood analysis, chest $\mathrm{x}$-ray, electrocardiography, echocardiography and indirect blood pressure (BP) measurement ${ }^{\mathrm{a}}$. On the following day, the dogs were anaesthetised for echocardiographic, CMRI and CCT examination using a standard anaesthesia protocol. After induction with levomethadon ${ }^{\mathrm{b}}(0.2 \mathrm{mg} / \mathrm{kg}$, IV $)$ and diazepam ${ }^{\mathrm{c}}(0.5 \mathrm{mg} / \mathrm{kg}, \mathrm{IV})$, Propofol ${ }^{\mathrm{d}}$ (IV) was administered intravenously until endotracheal intubation was possible in a dose up to $0.5 \mathrm{ml} / \mathrm{kg}$. Anaesthesia was maintained with isoflurane $e^{\mathrm{e}}(1.5 \%)$ in oxygen. First echocardiography was performed in the anteroom. Due to the lack of an automatic ventilation system in this room, the patient had to be ventilated manually, then CMRI and after that CCT were performed, where the dogs had to be ventilated continuously. The rate of breathing was approximately 12 breaths per minute regardless of being spontaneous, manual or continuous. Throughout the study $0.9 \%$ saline was infused at a rate of $3 \mathrm{ml} / \mathrm{kg} / \mathrm{h}$. BP was measured during anaesthesia. The duration of anaesthesia from induction till the end of CMRI was 120 minutes.

\section{DE}

All echocardiographical examinations were performed using a special heart ultrasound unit ${ }^{\mathrm{f}}$ with a V3 matrixarray probe $(1.5-4 \mathrm{MHz})$ for $3 \mathrm{DE}$ imaging. The probe contains more than 3000 piezocrystals. To record the ECG simultaneously during ultrasound examination, two ECG-electrodes were attached to the skin, one behind the right elbow and the other proximal on the left knee. During examination, the dogs were positioned in left lateral position. For an optimal image quality, a grey scale second-harmonic imaging technique with adjustment of 
image contrast, frequency, depth and sector size was used. The probe was held on the thorax from left-apical showing the complete long-axis of the two ventricles and their associated atriums. In the following, the plane was optimised for the right ventricle by more cranial probe positioning. To avoid foreshortening of the right ventricle, care had to be taken that the top of the right ventricle was always perpendicular.

In the 4D mode, seven slices were chosen for depicting the right ventricle, which consisted of three axial planes (Figure 1) and four short-axis slices. The first axial plane showed a four-chamber view, the second a two-chamber view (right ventricle and atrium) and the third a three chamber view (inflow- and outflow-tract). The three planes were at angles of approximately $60^{\circ}$ to each other. At least, the residual four short-axis slices reached from the apex to the atrioventricular ring. Volume data sets were acquired by single-beat examination with a mean frame rate of 16.8 ( $\min -\max =8.7$ to 20$)$ frames $/ \mathrm{s}$ (fps). During data acquisition care had to be taken that all right ventricular structures were clearly visible in all recorded planes. All echocardiographical examinations were performed by the same experienced cardiologist (S.O.H.).

\section{Quantification of RV volumes}

The RV volume 3D data sets were evaluated offline with special software developed for RV quantification ${ }^{\mathrm{g}}$. Sagittal, four-chamber and coronal views from one cardiac cycle were loaded into the RV-volume analysis window. After optimal alignment of the RV, markers were placed in the centre of the tricuspid and mitral valve as well as at the LV apex. In the following, initial contours were adjusted in the four-chamber, sagittal and coronal view by manually tracing lines along the RV chamber boundaries. As in the other methods inflow and outflow tract as well as papillary muscles, moderator bands and trabeculae were included in the RV volume [23]. By means of the contour revision function and using the highest sensitivity of contour identification, an entire RV volume set was generated and computed throughout the complete cardiac cycle (Figure 2). Maximum volume was defined as end-diastolic volume (EDV) and minimum volume as end-systolic volume (ESV) as in the other implemented methods. Based on a software integrated physic modelling algorithm, the EDV and ESV were assessed. Stroke volume (SV) was defined as the difference between EDV and ESV and ejection fraction (EF) as the percentage change of these volumes.

\section{CMR imaging}

The CMRI examination was performed with a 3.0 Tesla MRI $^{\mathrm{h}}$. Four MRI-compatible ECG electrodes ${ }^{\mathrm{i}}$ were stuck on the shaved skin at the left chest wall by positioning the first three at a right angle to each other and the last one slightly caudal of them. In supine position, four surface coils ${ }^{j}$ were placed in an overlapping technique dorsal and ventral on the thorax. To avoid motion artifacts, scans were recorded during end-expiratory breath-holds.

For scanning, a fast field echo (FFE) sequence was used with the following parameters: TE (time to echo) $2 \mathrm{~ms}$; TR (time to repeat) $4 \mathrm{~ms}$, flip angle $40^{\circ}$; matrix $256 \times 256$ and pixel size $1.2 \times 1.2$. Image acquisition took place with dedicated scan software ${ }^{\mathrm{k}}$. The scans started with compiling survey images of the cardiac region in sagittal, transversal and coronal orientation. From these survey images a short-axis stack was planned, which was adjusted to be parallel to the tricuspid valve and perpendicular to the septum. During several end-expiratory breath-holds, shortaxis slices covering the entire ventricle from heart base to apex were acquired with a slice thickness of $4.0 \mathrm{~mm}$ and without a slice gap. Each acquired image stack consisted of 18-22 slices, whereas each slice contained 30 heart phases.

After image acquisition, the evaluation of RV volume was accomplished by means of the disc summation method. Contours were drawn manually along the

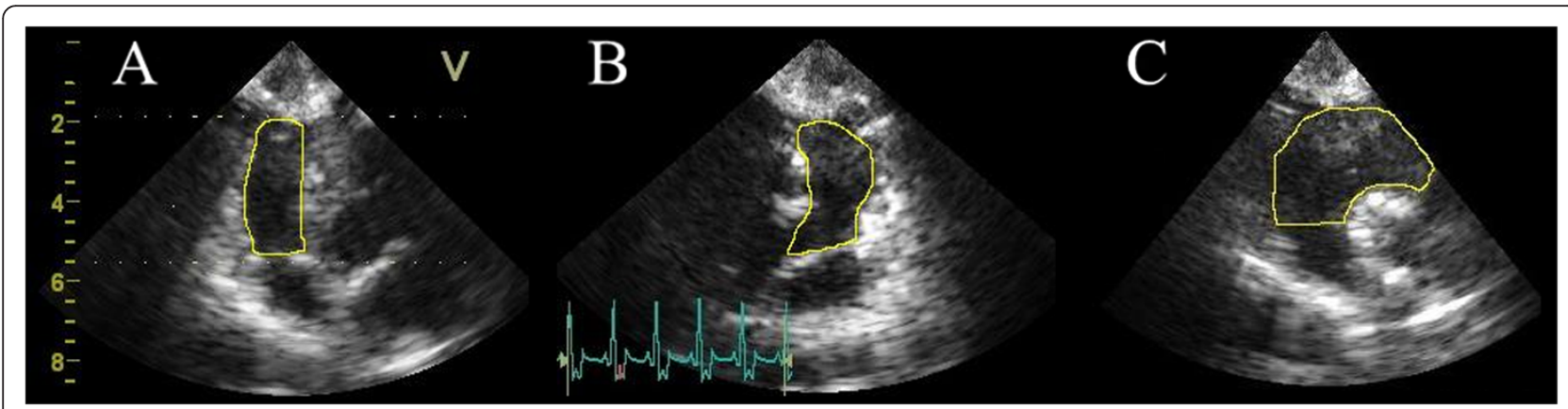

Figure 1 Three-dimensional echocardiography (3DE) of the right ventricle in an anaesthetised healthy beagle. The right ventricle is shown in three planes which are $60^{\circ}$ to each other: The four-chamber (A), two chamber (B) and three chamber view (C). The endocardial contours (yellow line) are manually drawn for better identification and as in all performed methods the papillary muscles and trabeculae were included in the volumes. 


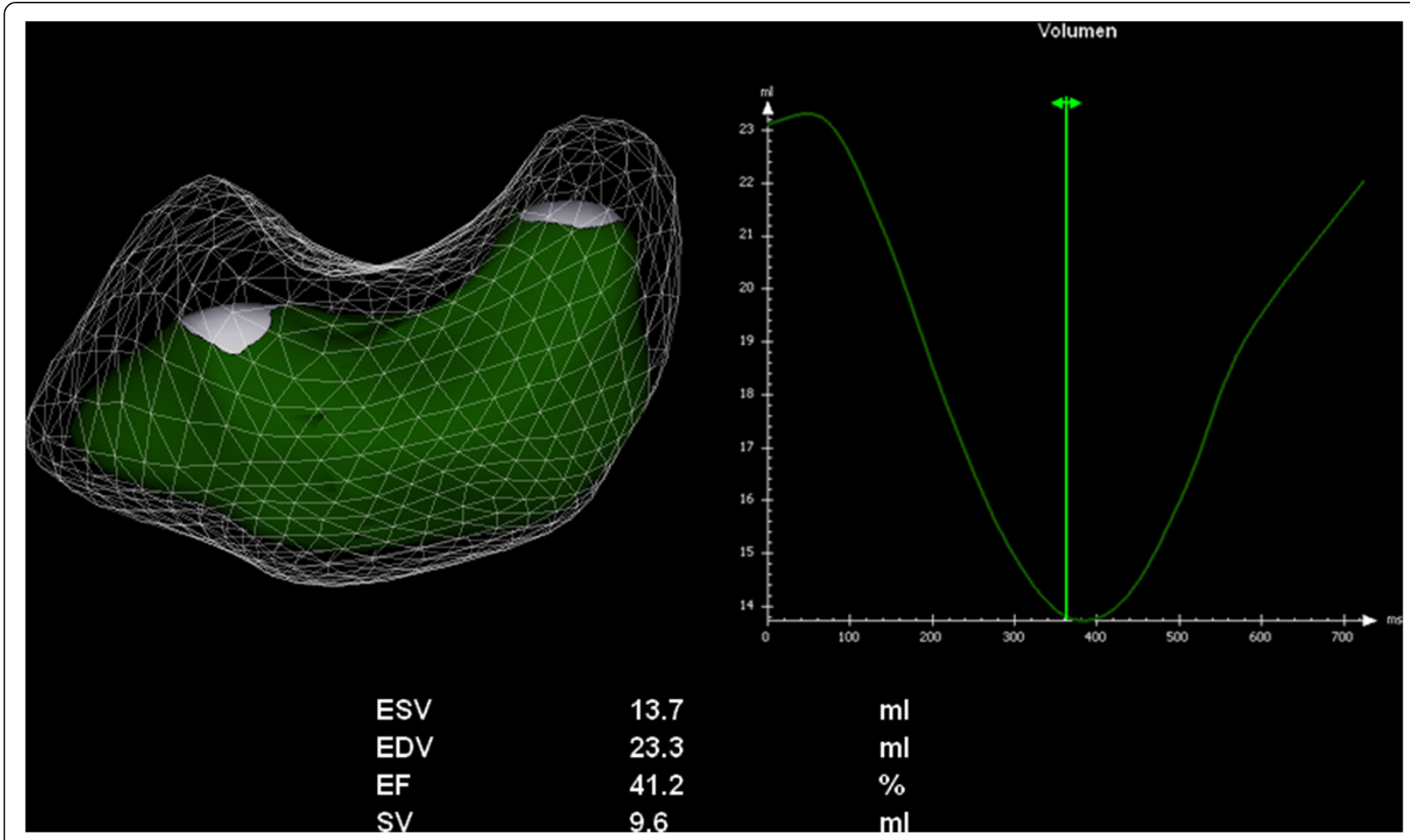

Figure 2 Results of three-dimensional echocardiography right ventricular function analysis of an anaesthetised healthy beagle. The model of the RV surface shows the end-diastolic volume (EDV) as transparent volume, whereas the end-systolic volume (ESV) is shown in green. The volume-time curve (VTC) demonstrates the changes of right ventricular volume ( $y$-axis) over time ( $x$-axis). Automatically minimal volume (ESV) and maximal volume (EDV) as well as ejection fraction and stroke volume are calculated from this VTC.

endocardial boundaries from the apex to the base of the right ventricle in end-diastolic and end-systolic images (Figure 3). The end-diastolic image was defined as the frame with maximum dilatation and the end-systolic image as the frame with maximum contraction. The first slice with a visible RV lumen was identified as the apex. In the basal slice, when the pulmonary valve was visible, contours were drawn up to the junction with the valves [24] because the RV outflow tract should be included in the measurements. Moreover, papillary muscles and trabeculae were attributed to the RV volume, whereas the interventricular septum, epicardial fat, and the pericardium were excluded. For determining EDV and ESV the disk summation method was used [25]. SV and EF were calculated as in 3DE.

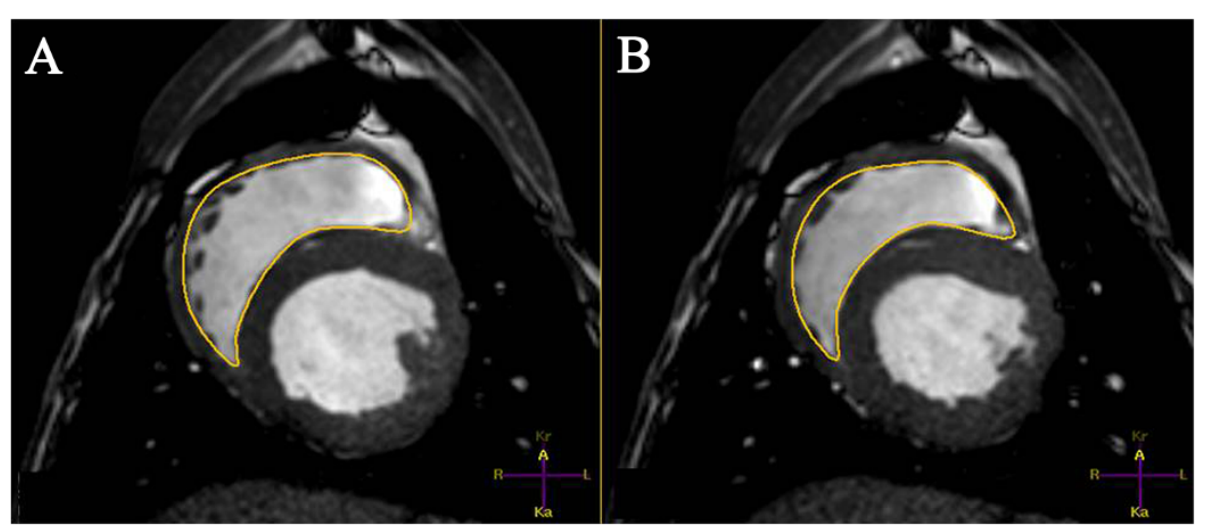

Figure 3 Cardiac magnetic resonance imaging (CMRI) right ventricular analysis of an anaesthetised healthy beagle. CMRI-short-axis is shown in (A) end-diastolic and (B) end-systolic state. The endocardial contours are manually drawn and as in all performed methods the papillary muscles and trabeculae were included in the volumes. 


\section{CCT examination}

CCT images were obtained by using a 64-detector-row $\mathrm{CT}^{1}$ with a gantry rotation time of $400 \mathrm{~ms}$, tube current of $400 \mathrm{~mA}$, tube voltage of $120 \mathrm{kV}$, detector collimation of $64 \times 0.625 \mathrm{~mm}$ and a table pitch of 0.20 . The dogs were placed in supine position and three ECG electrodes were applied to both forepaws and to the left hind paw for simultaneous ECG-recording during the scans. Scans were also performed during apnea.

At first, survey images from thorax entrance to diaphragm were created in sagittal and dorsal orientation in which the region of interest (ROI), the heart, was adjusted. Additionally, a small ROI was positioned in the ascending aorta for an automated bolus tracking technique [26]. For this purpose, an iodinated contrast medium ${ }^{\mathrm{m}}(2 \mathrm{ml} / \mathrm{kg}$, IV) was administered using a power injector ${ }^{\mathrm{n}}$ into a peripheral vein and the density of Hounsfield units (HU) was measured in the ROI of the aorta. When the threshold of 110 $\mathrm{HU}$ and an additional delay of $3.3 \mathrm{sec}$ had been exceeded, the helical scan started automatically.

Retrospectively, the spiral CT datasets were reconstructed in $10 \%$ steps throughout the cardiac cycle using a multi-segmental reconstruction algorithm. Thus, 10 heart phases emerged, which were shown as multiplanar reconstructions (MPR) with $0.9 \mathrm{~mm}$ slice thickness, $0.45 \mathrm{~mm}$ increment, $512 \times 512$ reconstruction matrix and an individual field of view.

Data analysis was accomplished in the Extended Brilliance Workspace ${ }^{\circ}$. In order to create short-axis images, the MPRs were loaded in a Cardiac viewer ${ }^{\mathrm{p}}$ with multiplanar view modus that showed three planes of the heart: the vertical long-axis, the horizontal longaxis and the short-axis plane. Axes had to be corrected manually. The short-axis reformations were generated in both long-axes planes parallel to the tricuspid valve and perpendicular to the septum. Phases with maximum dilatation and maximum contraction were defined as enddiastole and end-systole (Figure 4). Last of all, short-axis stacks were produced covering the entire right ventricle with 16 slices, a slice thickness of $3.0 \mathrm{~mm}$ and an interslice gap of $3.7-4.1 \mathrm{~mm}$ (min - max). For evaluating the RV volume, the disk summation method was used. For this, the end-diastolic and the end-systolic short-axis stacks were loaded into the LV/RV Analysis software ${ }^{\mathrm{q}}$. The same conditions for slice selection were applied as for CMRI. Despite the use of semi-automated border detection, manual correction was necessary.

\section{Statistical analysis}

For statistical tests and graphic presentation special software $^{r}$ was used. Heart rates and values of EDV, ESV, SV and EF were expressed as mean, median and standard deviation. CMRI and CCT data were measured once, whereas 3DE data were calculated as the mean of three heart cycles. To verify normal distribution of data, a Shapiro-Wilk test was performed. In the following, a single factor variance analysis was used to test the null hypothesis that CCT and 3DE programs provide identical results when measuring LV volumes and function compared to CMRI. Multiple pairwise comparisons between the analysing methods were performed to examine whether significant differences occur. A p-value of $<0.05$ was considered significant. For each pair of values, limits of agreement and systematic errors were assessed by evaluating the mean difference (bias) and the standard deviation of the differences using the Bland-Altman method. The strength of relation between each analysing technique and CMRI reference values was expressed by linear regression analysis with Spearman correlation coefficients. Correlations were defined as excellent with $R \geq 0.90$, very good with $R \geq 0.70$ and $<0.90$, less good with $R \geq 0.50$ and $<0.70$ and weak with $R<0.50$.

The reproducibility of 3DE data was tested by reevaluating the first analysis by the main investigator (A.K.S.) more than 2 weeks after the first measurement. Relative differences, correlation and significances were calculated for each parameter. To assess the variability, correlation and significances between two observers, the same blinded data were evaluated by a different observer (S.O.H.). The intra-observer and inter-observer variability were expressed as relative difference (percentages), defined as difference of the means between the two measurements, divided by their mean value and multiplied by 100 .

\section{Results}

All investigated dogs were clinically healthy with blood values within normal range. They had a regular sinus rhythm and their vertebral heart scales ranged from 10.0 to 11.2 with a mean of 10.5 . Their mean systolic BP was $153.3 \pm 13.8 \mathrm{mmHg}$ and their mean diastolic BP was $88.1 \pm 13.5 \mathrm{mmHg}$. During echocardiographical examination in four dogs minimal mitral valve regurgitation was found with a reflux smaller than $20 \%$ of the left atrium using colour Doppler, and with a broken profile in early systole using continuous-wave Doppler.

In anaesthesia, dogs had a mean systolic BP of $112.3 \pm$ $13.21 \mathrm{mmHg}$ and a mean diastolic BP of $57 \pm$ $14.84 \mathrm{mmHg}$. The heart rates were not significantly different between the different methods (echocardiographical examination: $96.3 \pm 14.33$ beats per minute (bpm), CCT: $95.4 \pm 11.96 \mathrm{bpm}$, CMRI $89.8 \pm 9.11 \mathrm{bpm})$.

All examinations were performed without complications. The landmarks RV cavity, RV wall, RV outflow tract (infundibulum, body), tricuspid and pulmonary valve could be found in all image data sets. However, image quality differed between the three image modalities. In the CMRI images all named structures were 


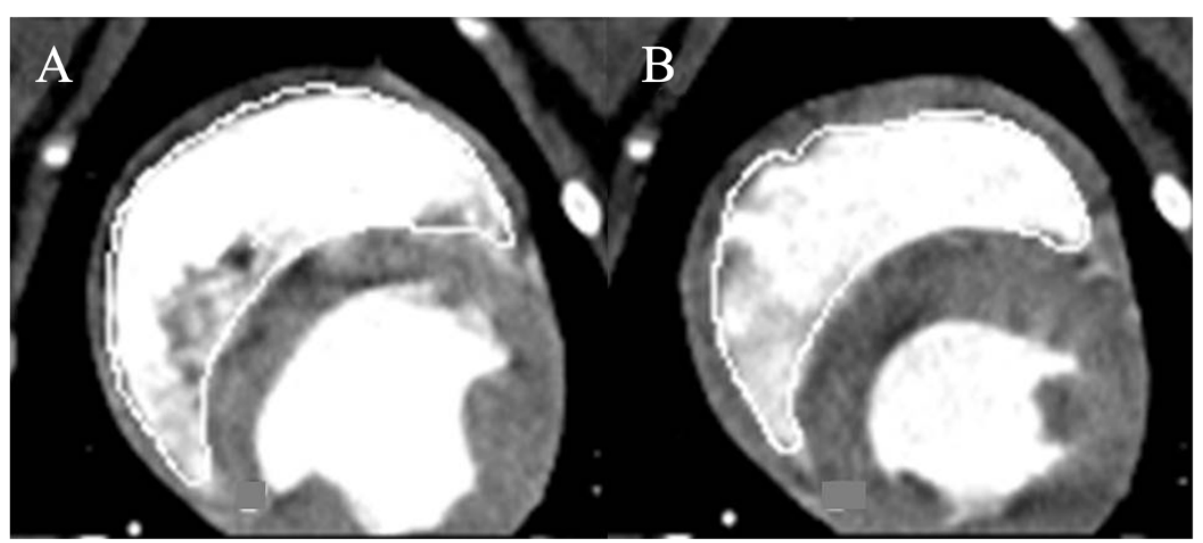

Figure 4 Midventricular short-axis of the right ventricle in cardiac computed tomography (CCT) of an anaesthetised healthy beagle. In CCT-short-axis the endocardial border is manually traced in (A) end-diastolic and (B) end-systolic volume and as in all performed methods the papillary muscles and trabeculae were included in the volumes.

clearly visible, except tricuspid and pulmonary valves which were more difficult to identify. CCT images were also of good quality, but the RV wall and the valves were slightly more difficult to define. The visualisation of the RV outflow tract was limited in all 3DE data sets. Median, mean \pm SD of CMRI, CCT and 3DE examinations are summarised in Table 1, the results of correlation and Bland-Altman analysis in Table 2 and Figures 5 and 6.

\section{Values of end-diastolic and end-systolic volume}

When comparing with CMRI, 3DE underestimated (bias $\pm \mathrm{SD}: \mathrm{EDV}=22.09 \pm 3.33, \mathrm{ESV}=15.07 \pm 3.94)$ the RV volumes significantly (EDV and ESV: $p<0.0001$ ), whereas CCT showed significant (EDV: $\mathrm{p}=0.0084$, ESV: $p=0.00128)$ different values with slightly higher volumes (bias $\pm \mathrm{SD}$ : $\mathrm{EDV}=-5.02 \pm 4.73, \mathrm{ESV}=-3.9 \pm$ $3.98)$, both methods presenting very good to excellent correlations (EDV: CCT: $\mathrm{R}=0.88,3 \mathrm{DE}: \mathrm{R}=0.96$; $\mathrm{ESV}=$ CCT: $\mathrm{R}=0.93$, 3DE: $\mathrm{R}=0.82$ ).

\section{Values of stroke volume}

SV was not different $(\mathrm{p}=0.1896)$ between CMRI and CCT (SV [Median \pm SD]: CMR $=19.3 \pm 1.9 \mathrm{ml}$; CCT: $20.4 \pm 1.8 \mathrm{ml})$ with little bias $(-1.1 \pm 2.47 \mathrm{ml})$ and weak correlation $(\mathrm{R}=0.18)$. When comparing CMRI and 3DE the SV was significantly $(\mathrm{p}=<0.0001)$ lower using 3DE (SV: $12.2 \pm 2.9)$ with wide bias $(7.05 \pm 3.11)$ and weak correlation $(\mathrm{R}=0.20)$.

\section{Values of ejection fraction}

In comparison to CMRI, EF was significantly $(\mathrm{p}=0.045)$ overestimated (bias \pm SD: $-7.34 \pm 6.17$ ) by $3 \mathrm{DE}$ with less good correlation $(\mathrm{R}=0.68)$, whereas using CCT EF was not significantly different (bias $\pm \mathrm{SD}: 1.59 \pm 4.35, \quad \mathrm{P}=$ $0.2771)$ with very good correlation $(R=0.71)$.

\section{Reproducibility of 3DE data}

For the RV data of 3DE, the relative differences between two observers were found to be $11 \%$ for EDV, $13 \%$ for ESV, 9\% for SV and 1\% for EF (Table 3), respectively with little but significant difference only for ESV and very good correlation $(\mathrm{R}>0.7)$ for EDV and ESV, and less good correlations for $E F(R=0.58)$ and $S V(R=0.26)$. The relative differences between measurements of the same observer were $2 \%$ for $\mathrm{EDV}, 3 \%$ for $\mathrm{ESV}, 0 \%$ for SV and $1 \%$ for $\mathrm{EF}$ (Table 4), respectively without significant differences and very good correlation for EDV, ESV and EF $(\mathrm{R}>0.81)$ and less good correlation for SV $(\mathrm{R}=0.58)$.

Table 1 Right ventricular function variables

\begin{tabular}{llll}
\hline Variable & CMRI & CCT & 3DE \\
\hline EDV $(\mathrm{ml})$ & $47.73 \pm 6.51(47.46)^{\mathrm{a}, \mathrm{b}}$ & $52.75 \pm 9.83(50.05)^{\mathrm{a}, \mathrm{c}}$ & $25.64 \pm 6.87(26.20)^{\mathrm{b}, \mathrm{c}}$ \\
ESV $(\mathrm{ml})$ & $28.45 \pm 7.13(27.46)^{\mathrm{a}, \mathrm{b}}$ & $32.35 \pm 9.45(30.70)^{\mathrm{a}, \mathrm{c}}$ & $13.38 \pm 4.86(13.18)^{\mathrm{b}, \mathrm{c}}$ \\
$\mathrm{SV}(\mathrm{ml})$ & $19.27 \pm 1.91(19.50)^{\mathrm{b}}$ & $20.38 \pm 1.78(20.15)^{\mathrm{c}}$ & $12.22 \pm 2.89(12.40)^{\mathrm{b}, \mathrm{c}}$ \\
EF $(\%)$ & $41.13 \pm 7.21(40.80)^{\mathrm{b}}$ & $39.54 \pm 6.04(41.10)^{c}$ & $48.47 \pm 7.13(50.25)^{\mathrm{b}, \mathrm{c}}$ \\
\hline
\end{tabular}

Mean \pm standard deviation values for right ventricular RV function variables (end-diastolic volume $=\mathrm{EDV}$, end-systolic volume $=\mathrm{ESV}$, stroke volume $=\mathrm{SV}$, ejection fraction $=E F$ ) obtained with cardiac magnetic resonance imaging (CMRI), cardiac computed tomography (CCT) and three-dimensional echocardiography (3DE) in 10 healthy anaesthetised beagles.

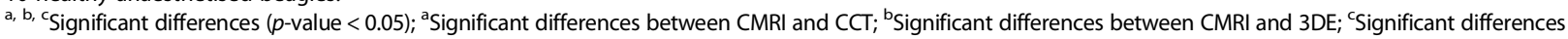
between CCT and 3DE. 
Table 2 Statistical evaluation of different methods of right ventricular volume quantification

\begin{tabular}{|c|c|c|c|c|c|c|}
\hline \multirow[b]{2}{*}{ Variable } & \multirow[b]{2}{*}{ Techniques compared } & \multirow{2}{*}{$\begin{array}{l}\text { Group } \\
\text { Comparison }\end{array}$} & \multicolumn{2}{|c|}{ Correlation } & \multicolumn{2}{|c|}{ Bland-Altman analysis } \\
\hline & & & $\mathrm{R}$ & $P$ value & Bias & SD \\
\hline & CMRlvs 3DE & $<0.0001^{a}$ & 0.96 & $<0.0001^{a}$ & 22.09 & 3.33 \\
\hline \multirow[t]{3}{*}{ EDV } & CMRIvs CCT & 0.0084 & 0.88 & $0.0009^{\mathrm{a}}$ & -5.02 & 4.73 \\
\hline & $C C T$ vs 3DE & $<0.0001^{\mathrm{a}}$ & 0.80 & 0.0052 & 27.11 & 5.69 \\
\hline & CMRIvs 3DE & $<0.0001^{\mathrm{a}}$ & 0.82 & $0.0038^{\mathrm{a}}$ & 15.07 & 3.94 \\
\hline \multirow[t]{3}{*}{ ESV } & CMRIvs CCT & $0.0128^{\mathrm{a}}$ & 0.93 & $0.0001^{\mathrm{a}}$ & -3.9 & 3.98 \\
\hline & CCT vs 3DE & $<0.0001^{a}$ & 0.88 & $0.0008^{\mathrm{a}}$ & 18.97 & 5.67 \\
\hline & CMRlvs 3DE & $<0.0001^{\mathrm{a}}$ & 0.20 & 0.5784 & 7.05 & 3.11 \\
\hline \multirow[t]{3}{*}{ SV } & CMRlvs CCT & 0.1896 & 0.18 & 0.6130 & -1.11 & 2.47 \\
\hline & $C C T$ vs $3 D E$ & $<0.0001^{\mathrm{a}}$ & 0.10 & 0.7763 & 8.16 & 3.34 \\
\hline & CMRlvs 3DE & $0.0045^{\mathrm{a}}$ & 0.68 & $0.0289^{\mathrm{a}}$ & -7.34 & 6.17 \\
\hline \multirow[t]{2}{*}{ EF } & CMRIvs CCT & 0.2771 & 0.71 & $0.0217^{\mathrm{a}}$ & 1.59 & 4.35 \\
\hline & $C C T$ vs 3DE & $0.0015^{\mathrm{a}}$ & 0.72 & $0.0186^{\mathrm{a}}$ & -8.93 & 6.28 \\
\hline
\end{tabular}

Group comparison (GC), correlation coefficients and Bland-Altman analysis (bias and standard deviation [SD]) for values of right ventricular function (end-diastolic volume $=\mathrm{EDV}$, end-systolic volume $=\mathrm{ESV}$, stroke volume $=\mathrm{SV}$, ejection fraction $=\mathrm{EF})$ obtained with cardiac magnetic resonance imaging $(\mathrm{CMRI})$, cardiac computed tomography (CCT) and three-dimensional echocardiography (3DE) in 10 healthy anaesthetised beagles.

a Significant differences $(p<0.05)$.

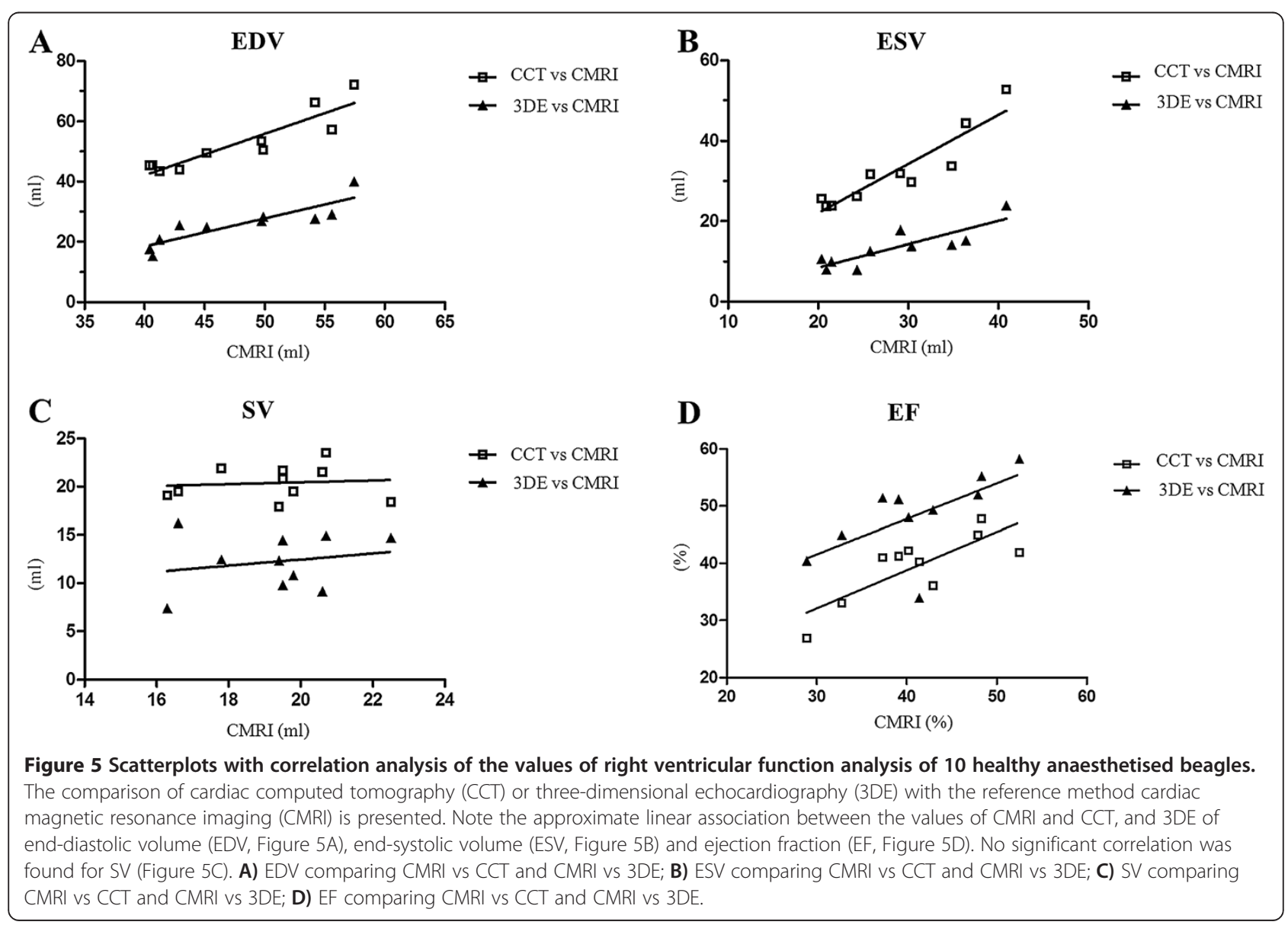



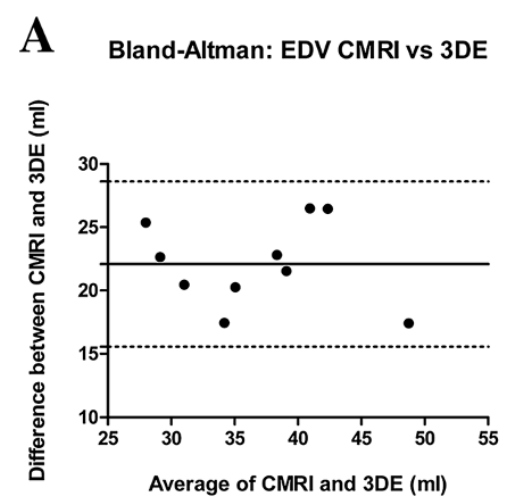

C Bland-Altman: ESV CMRI vs 3DE

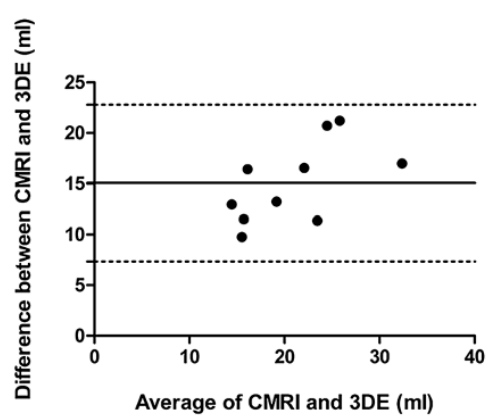

E Bland-Altman: SV CMRI vs 3DE
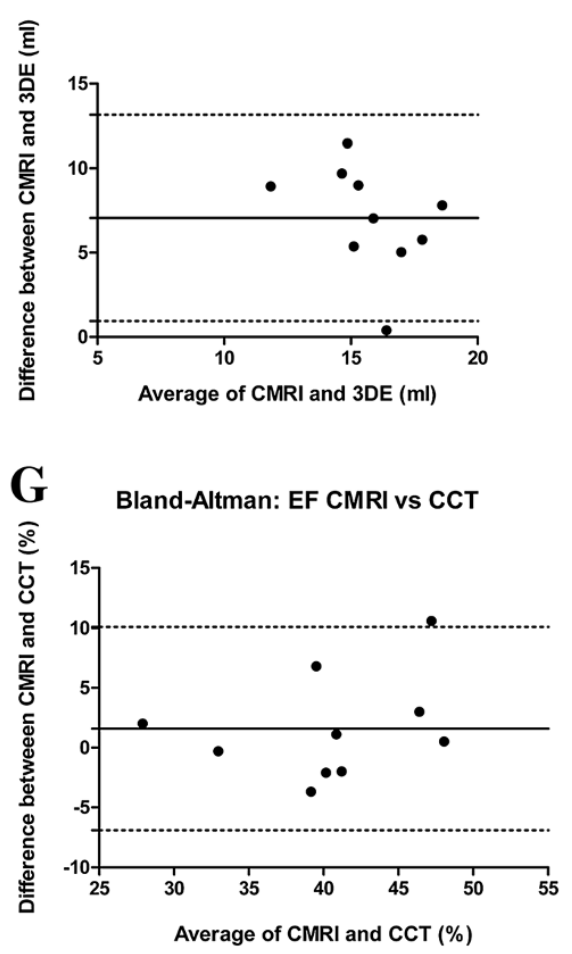

B Bland-Altman: EDV CMRI vs CCT

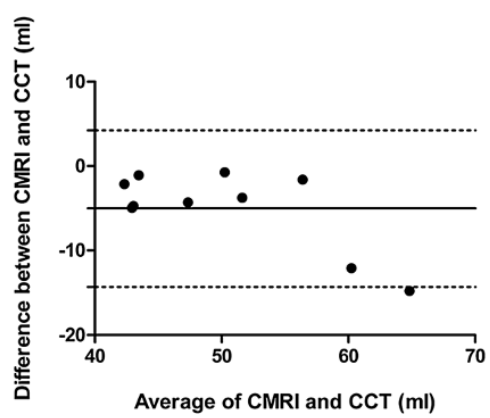

D Bland-Altman: ESV CMRI vs CCT

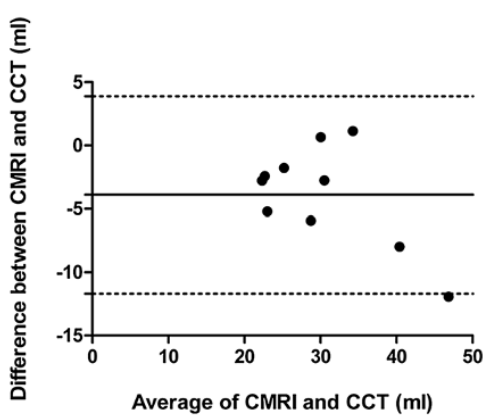

F Bland-Altman: SV CMRI vs CCT

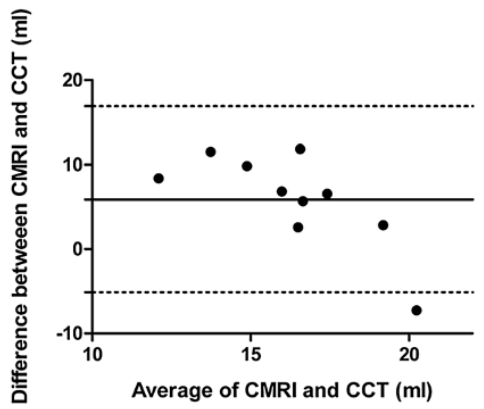

H Bland-Altman: EF CMRI vs 3DE

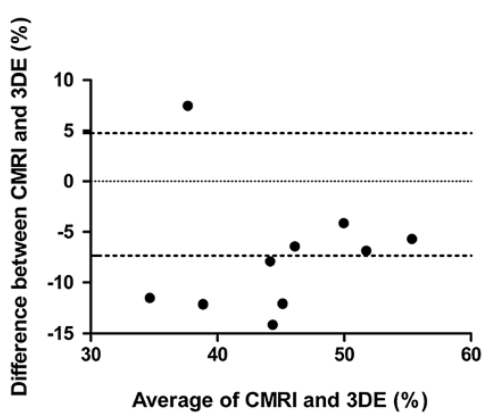

Figure 6 (See legend on next page.) 
(See figure on previous page.)

Figure 6 Bland-Altman plots of the values of right ventricular function analysis of 10 healthy anaesthetised beagles. The comparison of cardiac computed tomography (CCT) or three-dimensional echocardiography (3DE) with the reference method cardiac magnetic resonance imaging (CMRI) is presented. In each panel, the solid horizontal line represents the mean value of differences (bias) and the dotted lines the limits of agreement (bias \pm 1.96 standard deviation). Note that there is an only small bias between CCT and CMRI as opposed to the large bias between $3 D E$ and $C M R I$ (end-diastolic volume $=E D V$, end-systolic volume $=E S V$, ejection fraction $=E F$, stroke volume $=$ SV). A) EDV comparing CMRI vs 3DE; B) EDV comparing CMRI vs CCT; C) ESV comparing CMRI vs 3DE; D) ESV comparing CMRI vs CCT; E) SV comparing CMRI vs 3DE; F) SV comparing CMRI vs CCT; G) EF comparing CMRI vs 3DE; H) EF comparing CMRI vs CCT.

\section{Discussion}

In the present study, the right ventricles of 10 Beagle dogs were investigated by means of $3 \mathrm{DE}$ and CCT in comparison with the reference method CMRI. The major challenge for determining right ventricular volume is the right ventricle itself. The complex shape with its segmentation in body, inflow and outflow tract, the thin RV wall and the heavy trabeculation make the RV measurement in twodimensional echocardiography difficult [6]. CMRI is the gold standard for RV volumetric quantification in human medicine [18] and overcomes the limitation of 2D echocardiography by using a three-dimensional volume set, as well as the 3D modalities 3DE and CCT. Although the assessment of left ventricular function using two- and threedimensional volume estimation is part of various veterinary publications [27-30], there are to the best knowledge of the author no veterinary studies concerning the evaluation of right ventricular function either by means of twodimensional volume calculation or even three-dimensional volume measurement. Comparing the methods in this study, 3DE would be the most practical in veterinary cardiology, reasoned by the unnecessity for anaesthesia and the inexpensive hardware. The focus of this study was the comparison of 3DE, CCT and CMRI under as comparable conditions as possible; therefore all measurements were performed in anaesthesia. The evaluation of the usefulness of 3DE in awake dogs will be part of further studies. CT scanners in contrast with MRI scanners are nowadays quite common in the veterinary clinic. Therefore, its usefulness for RV volumetric measurement was investigated as well.

The results of the current study showed significant lower RV volumes by using 3DE and significant higher RV volumes by using CCT compared with CMRI with very good to excellent correlations. Differences between the modalities can have various reasons, which are in context with their different image quality, data acquisition and reconstruction, temporal resolution, selection of the last basal slice and the use of contrast agent for CCT examination.

This study was performed in general anaesthesia, whereas human studies have been performed in conscious patients. One can speculate that the negative inotropic effect caused by isofluran [31] results in higher RV volumes (especially ESV) and lower SV and EF the gravest in the last performed modality (CCT) with the longest duration of anaesthesia. Since 3DE was performed as first examination and showed the lowest RV volumes, whereas CCT was performed last with the highest RV volumes, an anaesthesia associated effect cannot be completely ruled out.

Furthermore, different patient positioning could have influenced the results of this study. A lower ventricular filling in supine position with lower stroke volume is described by catheter intervention or thermodilution technique [32-34] in narcotised animals. This is contrary to our results because the lowest RV-volumes exist for 3DE measurements in lateral recumbency, whereas the other modalities with higher volumes were performed in supine position. Therefore, this possible positioning associated effect is probably concealed by the methodical difference caused by the various techniques.

Furthermore, the differing ventilation protocol could have possibly influenced the results in this study because CCT and CMRI were performed in apnoea and 3DE was not. However, we took care not to acquire 3DE volume data sets during breathing. Therefore, this influence could not have been that intense.

In human medicine, the 3DE underestimation of RV volumes compared with CMRI (and CCT) is well known [9]. However, in contrast to our study, the investigations

Table 3 Inter-observer variability of three-dimensional echocardiography

\begin{tabular}{llllll}
\hline Variable & First observer $(\mathbf{m l})$ & Second observer $(\mathbf{m l})$ & Relative difference (\%) & Group comparison (P-Values) & Correlation coefficient (R) \\
\hline EDV & 25.64 & 28.61 & 10.94 & 0.160 & 0.71 \\
ESV & 13.38 & 15.20 & 12.74 & $0.027^{\mathrm{a}}$ & 0.83 \\
SV & 12.22 & 13.42 & 9.40 & 0.556 & 0.26 \\
EF & 48.47 & 47.99 & 1.00 & 0.695 & 0.58 \\
\hline
\end{tabular}

Comparison of right ventricular function parameters measured with three-dimensional echocardiography by two different observers (end-diastolic volume = EDV, end-systolic volume $=E S V$, ejection fraction $=E F$, stroke volume $=S V$ ).

${ }^{a}$ Significant differences $(p<0.05)$. 
Table 4 Intra-observer variability of three-dimensional echocardiography

\begin{tabular}{llllll}
\hline Variable & $\begin{array}{l}\text { First } \\
\text { measurement }(\mathbf{m l})\end{array}$ & $\begin{array}{l}\text { Second } \\
\text { measurement }(\mathbf{m l})\end{array}$ & $\begin{array}{l}\text { Relative } \\
\text { difference (\%) }\end{array}$ & $\begin{array}{l}\text { Group comparison } \\
(\text { P-Values) }\end{array}$ & $\begin{array}{l}\text { Correlation } \\
\text { coefficient (R) }\end{array}$ \\
\hline EDV & 25.64 & 26.05 & 1.60 & 0.695 & 0.81 \\
ESV & 13.38 & 13.84 & 3.45 & 0.625 & 0.95 \\
SV & 12.22 & 12.22 & 0.00 & 0.922 & 0.58 \\
EF & 48.47 & 48.09 & 0.80 & 0.846 & 0.84 \\
\hline
\end{tabular}

Comparison of right ventricular function parameters measured twice at least two weeks apart with three-dimensional echocardiography by the same observer (end-diastolic volume $=E D V$, end-systolic volume $=E S V$, ejection fraction $=E F$, stroke volume $=S V$ ).

were carried out in conscious human patients with cardiac disease, therefore the results are not directly comparable. In agreement with previous human medical studies $[6,9,19,23]$, we made the experience that several factors influence the measurements of RV cavity in 3DE. One factor is the confounding effect of the apical trabeculae on endocardial tracking $[6,9,23]$. Furthermore, the worse demarcation of the RV anterior free wall and the RV outflow tract from the surroundings and the indistinct visualisation of the pulmonary valve lead to difficulties in correct contour tracing [19]. Thus, the restricted image quality of $3 \mathrm{DE}$ may be the main reason for underestimation of the RV volume. On comparing Figures 1, 3 and 5 it is apparent that the border delineation with CMRI and CCT is far better than with 3DE. Perhaps contrast-enhanced 3D volumetric quantification could be a future aspect for better RV border detection, as shown in human medicine [35].

Another reason causing underestimation of RV volumes could be the low temporal resolution. As the definition of EDV and ESV in all performed methods was based on the maximum and minimum RV volume and not defined according to ECG the measurements were not necessarily carried out at exactly the same time point due to the differing temporal resolution. The lower the temporal resolution, the lower is the probability of detecting the maximum or minimum RV volume. Nevertheless, the definition of EDV and ESV according to ECG is imprecise since, for example, the RV maximum volume exists somewhere in the $\mathrm{R}$ wave.

In our study 3DE temporal resolution ranged from 8.7 to $20 \mathrm{fps}$. Compared with the temporal resolution of CMRI of $30 \mathrm{fps}$ this is rather low. A frame rate of $20-$ $40 \mathrm{fps}$ is considered sufficient in left ventricular $3 \mathrm{DE}$ volumetric quantification [36,37]. The technical problem of achieving a good temporal as well as spatial resolution is discussed intensively in human cardiology $[7,38,39]$. In $\mathrm{RV}$ volumetric measurement an extremely wide field of view is necessary, which causes low frame rates. Human studies used frame rates of 14 up to 40 fps by using $7-8$ subvolumes $[10,23,40]$. The ultrasound machine in the present study is able to generate single-beat volumes and we achieved a mean frame rate of $16.8 \mathrm{fps}$ for RV depiction. This is equivalent to approximately 10 volumes per heart cycle (heart rate $=96$ beats per minute), causing a low temporal resolution. Considering CCT, the temporal resolution was comparable to $3 \mathrm{DE}$ with 10 frames per heart cycle, whereas CMRI has a temporal resolution of 30 frames per heart cycle. By using 3DE with subvolumes of different heart cycles it is possible to generate higher frame rates, but the spatial resolution is worse. In this study the delineation of the RV endocardium was very difficult, using up to 4 subvolumes without stitching artifacts. In four cases it was not possible to obtain stitching artifactfree images. Consequently, the single-beat mode for RV volume evaluation was used. For precise measurements a high temporal resolution is needed [14]. Therefore, best results are expected with CMRI. Furthermore, the lower temporal resolution of CCT is described as a potential source of error in many publications [41,42]. The same applies to the low temporal resolution of 3DE. Thus, it could be another factor for underestimation of RV volume.

However, the higher RV volumes with CCT explained only by its limited temporal resolution alone is implausible. Although CCT could result in overestimated ESV by missing the smallest volume, it is not presumable that it leads to overestimated EDV [43]. It is more plausible that the overestimation using CCT is caused by the fast $(<20 \mathrm{sec})$ injection of a quite large volume $(2 \mathrm{ml} / \mathrm{kg})$ of contrast medium [43]. The iodine contrast solution arrives in the right ventricle immediately after injection causing higher RV volumes by increasing preload and minimal negative inotropic effects [43-45], whereas the concentration of the contrast medium in the left ventricle was less pronounced because of dispersion in the pulmonary circulation.

The method of data acquisition and evaluation differed in the three imaging modalities. While specific sectional planes have to be defined in CMRI during the scans and cannot be changed afterwards, CCT and 3DE generate modifiable 3D volume sets with optimisation of the sectional planes later on. The subsequent reconstruction of the data sets has the advantage of being more flexible: Data sets can be reconstructed and manipulated in $\mathrm{x}, \mathrm{y}, \mathrm{z}$ axes as often as necessary for correctly fitted short-axes [46]. Consequently, the risk of oblique short-axis slices arising is higher in CMRI because image adapting cannot be repeated after the examination. The selection of oblique 
short-axis slices would tend to overestimate the volume calculated from summated short-axis slices [47]. To avoid overestimation and also underestimation we included the area between the tricuspid and pulmonary valve near the heart base in CMRI and CCT short-axes. If only slices were included in which the right ventricle was a unit, the volumes would be underestimated in cases of oblique slices. Otherwise, volume would be overestimated if the tricuspid and the pulmonary valves would be ignored and the right atrium and pulmonary artery would be included in the volume. However, the assessment in the short-axis orientation complicates identification of the position of the pulmonary and tricuspid valves, which could also result in erroneous measurements [24]. Moreover, partial volume effects of the basal slice inherent in the short-axis method were a reason for wrong results [48]. Consequently, the determination of the last included slice near the heart base can be a source of error in cases of CCT and CMRI measurements.

For that reason, the 4D software of 3DE has the advantage of not using the short-axis alone but also with two axial planes for contour delineation during evaluation. This could be useful because the RV outflow tract and the pulmonary valve are orientated angular to the short-axis slices, which makes determination of RV contour complicated for CCT as well as for CMRI [41]. Nevertheless, because of its thin structure the pulmonary valve was often difficult to see in the coronal plan in 3DE, which resulted in impaired contour delineation. Consequently, the measurements were not improved by this technique in our study.

For evaluation of the RV volume and function, all three modalities used physic-based algorithms integrated in their software application that make no assumptions of RV geometry. Different quantification software used for CMRI, CCT and 3DE were described as a potential source of discrepancies [23]. In our study, the software RV-Volume ${ }^{\circ}$ (TomTec) was used to evaluate the 3DE data. In agreement with other publications, in which the same software was used, the RV volumes were underestimated [6]. However, the underestimation was not always significant $[9,10]$. Certainly, small inaccuracies in the software used are possible, but the main difference still exists because of the limited visualisation of the RV free wall.

The EF represents one of the most important functional parameters of the right ventricle. Impaired function results in inadequate SV and can lead to heart failure [3]. In our study we found similar results for EF and SV in CMRI and $\mathrm{CCT}$ values. Consequently, the function parameters are not affected by the method CMRI or CCT. A possible explanation for the significantly higher EF using $3 \mathrm{DE}$ is the more pronounced underestimation of the RV volume expressed as a percentage of CMRI in ESV (53\% of CMRI) than in EDV ( $46 \%$ of CMRI). Nevertheless, the possible negative inotropic effect of prolonged anaesthesia cannot be ruled out. As expected the SV was significantly lower using $3 \mathrm{DE}$ than in the other methods, caused by underestimation of the RV volumes.

CMRI is the gold standard for RV quantification in human medicine. Drawbacks of this technique are the necessity for anaesthesia, the long investigation time and the high costs. In cases of contraindication for CMRI (CMRI-incompatible implants), the CCT can be used. One reason for favouring the CCT investigation is the significant shorter data acquisition time. For the CCT investigation we needed 10 minutes, in contrast to the CMRI investigation which took approximately $50 \mathrm{mi}-$ nutes. The generation of acceptable 3DE RV volume data sets lasts about 10 minutes. On the contrary, limitation factors of CCT are the exposure of $\mathrm{x}$-radiation and the use of potentially nephrotoxic contrast medium.

The most non-invasive technique is the 3DE. Dogs can stay awake during examination, which is especially beneficial for dogs with heart failure because general anaesthesia is often complicated by the presence of cardiac disease [49]. It is possible that in awake dogs spatial resolution of $3 \mathrm{DE}$ is even worse, caused by the patient moving and panting. In this situation the single-beat quantification used in this study would be preferable because the multi-volume sample acquisition probably results in stitching artefacts. On the other hand, enlargement of the RV in dogs with right-sided heart disease demands even wider field of views so the temporal resolution has to be optimised, too. Therefore, the evaluation by 3DE in awake dogs with right-sided heart disease and differing body weight is an interesting topic for further studies.

A disadvantage of $3 \mathrm{DE}$ is the need for intensive and time-consuming manual border correction despite using the highest grade of sensitivity for automatic contour identification. The intra-observer variability was far better than inter-observer variability. This is caused by the difficult definition of the RV border and especially the pulmonic and tricuspid valve. Furthermore, the less good inter-observer variability is probably influenced by the use of slightly different contrast and echogenicity settings used during optimisation of the raw data sets by each observer. The intra-observer variability of lower $5 \%$ is good. Therefore, each observer could reliably identify the same borders in each repeated examination. This implies that measurements should possibly be performed by the same investigator in case of studies or repeated measurements.

\section{Limitations}

The main limitation of the study was the low number of study participants so that for general statements and creation of reference values studies with a larger number of participants (allocated into groups for gender, age and weight) are needed. Additionally, the influence of a 
prolonged anaesthesia and of differences in patient positioning as well as differences of ventilation technique cannot be ruled out completely. Moreover, in our study we investigated only dogs without relevant heart failure in anaesthesia. Therefore, further studies with dogs during wakefulness and dogs with right heart disease are needed to evaluate if early changes of the RV volume and function quantified with the $3 \mathrm{D}$ techniques can be used as a predictor of development of rightsided heart failure in dogs in the future. Further limitations of the study design were the low number of observers for the $3 \mathrm{DE}$ data and the limited imaging quality of 3DE data sets, as already mentioned. Probably, technical improvements, contrast echocardiography or multi-beat analysis will achieve better spatial and temporal resolution.

\section{Conclusion}

The excellent definition of endocardial borders and the high temporal resolution of CMRI images enable assessment of RV volume and function and confirm its use as gold standard. The CCT data sets also show well depiction but with significant overestimation of RV volumes, therefore these methods are not interchangeable. A good alternative for quantification of RV is the $3 \mathrm{DE}$ technique. Although 3DE values are not directly comparable with CCT and CMRI, caused by its underestimation of RV volumes, the excellent correlation let assume that $3 \mathrm{DE}$ is a feasible alternative.

\section{Endnotes}

${ }^{\mathrm{a}} \mathrm{VetHDO}^{\circ}$ High definition oscillometry, S + B MedVet GmbH, Germany

${ }^{\mathrm{b}} \mathrm{L}$-Polamivet ${ }^{\circ}$, IntervetGmBH, Germany

${ }^{\mathrm{c}}$ Diazepam-ratiopharm ${ }^{\circ}$, CP-Pharma GmBH, Germany

${ }^{\mathrm{d}}$ Narcofol $^{\circ}$, CP-Pharma GmBH, Germany

${ }^{\mathrm{e}}$ Isofluran ${ }^{\circ}$, CP-Pharma GmBH, Germany

${ }^{f}$ Vivid E9; GE healthcare, Germany

${ }^{\mathrm{g}}$ 4D RV-Volume; ${ }^{\circ}$ TomTec Imaging Systems $\mathrm{GmbH}$, Germany

${ }^{\mathrm{h}}$ Achieva 3.0 T; Philips, Netherlands

${ }^{\mathrm{i}}$ Radio-Translucent Foam Monitoring Electrodes, Germany

${ }^{j}$ Sense Flex Small/Medium; Philips, Netherlands

${ }^{\mathrm{k}}$ MR Systems Achieva, Philips, Netherlands

${ }^{\mathrm{l}}$ Brilliance 64; Philips, Netherlands

${ }^{\mathrm{m}}$ Xenetix $350 \mathrm{mg}$ Iod/ml, Guerbet GmBH, Germany

${ }^{\mathrm{n}}$ Medrad Vistron CT Injection System, Medrad, Pa.

${ }^{\circ}$ Extended Brilliance Workspace, Philips, Netherlands

${ }^{\mathrm{P}}$ Cardiac viewer, Philips, Netherlands

${ }^{\mathrm{q}}$ LV/RV Analysis software, Philips, Netherlands

${ }^{\mathrm{r}}$ SAS9.2, SAS Institute, Cary North Carolina, USA and Prism, GraphPad Software, La Jolla, USA.

\section{Abbreviations}

3DE: Three-dimensional echocardiography; CCT: Cardiac computed tomography; CMRI: Cardiac magnetic resonance imaging; ECG: Electrocardiogram; EDV: End-diastolic volume; EF: Ejection fraction; ESV: End-systolic volume; Fps: Frames per second; RV: Right ventricular; SV: Stroke volume; SD: Standard deviation.

\section{Competing interests}

The authors declare that they have no competing interests.

\section{Authors' contribution}

AKS, PW and $\mathrm{SOH}$ designed the study. AKS collected and analysed data, wrote the manuscript and made the figures. PD supported CCT and CMRI with data acquisition. SOH performed echocardiographical data acquisition and contributed to data analysis and interpretation. IN, PW and $\mathrm{SOH}$ helped with editing the manuscript. All authors read and approved the final manuscript.

\section{Acknowledgement}

The authors wish to thank Dr. Martin Beyerbach, Department of Biometry, Epidemiology and Information Processing, University of Veterinary Medicine, Hannover, Germany for his assistance with statistical analysis.

Received: 30 January 2014 Accepted: 24 September 2014

Published online: 12 October 2014

\section{References}

1. Mertens LL, Friedberg MK: Imaging the right ventricle-current state of the art. Nat Rev Cardiol 2010, 7(10):551-563.

2. Horton KD, Meece RW, Hill JC: Assessment of the right ventricle by echocardiography: a primer for cardiac sonographers. J Am SoC Echocardiogr 2009, 22(7):776-792. quiz 861-772.

3. Voelkel NF, Quaife RA, Leinwand LA, Barst RJ, McGoon MD, Meldrum DR, Dupuis J, Long CS, Rubin LJ, Smart FW, Suzuki YJ, Gladwin M, Denholm EM, Gail DB: Right ventricular function and failure: report of a National Heart, Lung, and Blood Institute working group on cellular and molecular mechanisms of right heart failure. Circulation 2006, 114(17):1883-1891.

4. Pariaut $R$, Saelinger $C$, Strickland KN, Beaufrere $H$, Reynolds CA, Vila J: Tricuspid Annular Plane Systolic Excursion (TAPSE) in dogs: reference values and impact of pulmonary hypertension. J Vet Intern Med 2012, 26(5):1148-1151.

5. Rudski LG, Lai WW, Afilalo J, Hua L, Handschumacher MD, Chandrasekaran K, Solomon SD, Louie EK, Schiller NB: Guidelines for the echocardiographic assessment of the right heart in adults: a report from the American Society of Echocardiography endorsed by the European Association of Echocardiography, a registered branch of the European Society of Cardiology, and the Canadian Society of Echocardiography. J Am Soc Echocardiogr 2010, 23(7):685-713. quiz 786-688.

6. Jenkins C, Chan J, Bricknell K, Strudwick M, Marwick TH: Reproducibility of right ventricular volumes and ejection fraction using real-time threedimensional echocardiography: comparison with cardiac MRI. Chest 2007, 131(6):1844-1851.

7. Lang RM, Badano LP, Tsang W, Adams DH, Agricola E, Buck T, Faletra FF, Franke A, Hung J, de Isla LP, Kamp O, Kasprzak JD, Lancellotti P, Marwick TH, McCulloch ML, Monaghan MJ, Nihoyannopoulos P, Pandian NG, Pellikka PA, Pepi M, Roberson DA, Shernan SK, Shirali GS, Sugeng L, Ten Cate FJ, Vannan MA, Zamorano JL, Zoghbi WA: EAE/ASE recommendations for image acquisition and display using three-dimensional echocardiography. Eur Heart J Cardiovasc Imaging 2012, 13(1):1-46.

8. Lang RM, Bierig M, Devereux RB, Flachskampf FA, Foster E, Pellikka PA, Picard MH, Roman MJ, Seward J, Shanewise J, Solomon S, Spencer KT, St John Sutton M, Stewart W: Recommendations for chamber quantification. Eur J Echocardiogr 2006, 7(2):79-108.

9. Gopal AS, Chukwu EO, Iwuchukwu CJ, Katz AS, Toole RS, Schapiro W, Reichek N: Normal values of right ventricular size and function by real-time 3-dimensional echocardiography: comparison with cardiac magnetic resonance imaging. J Am Soc Echocardiogr 2007, 20(5):445-455.

10. van der Zwaan HB, Geleijnse ML, McGhie JS, Boersma E, Helbing WA, Meijboom FJ, Roos-Hesselink JW: Right ventricular quantification in clinical practice: two-dimensional vs. three-dimensional echocardiography 
compared with cardiac magnetic resonance imaging. Eur J Echocardiogr 2011, 12(9):656-664.

11. Chen $G$, Sun $K$, Huang $G$ : In vitro validation of right ventricular volume and mass measurement by real-time three-dimensional echocardiography. Echocardiography 2006, 23(5):395-399.

12. Jiang L, Siu SC, Handschumacher MD, Luis Guererro J, de Prada JA V, King ME, Picard MH, Weyman AE, Levine RA: Three-dimensional echocardiography. In vivo validation for right ventricular volume and function. Circulation 1994, 89(5):2342-2350.

13. Juergens KU, Grude M, Maintz D, Fallenberg EM, Wichter T, Heindel W, Fischbach R: Multi-detector row CT of left ventricular function with dedicated analysis software versus MR imaging: initial experience. Radiology 2004, 230(2):403-410.

14. Mahnken AH, Spuentrup E, Niethammer M, Buecker A, Boese J, Wildberger JE, Flohr T, Sinha AM, Krombach GA, Gunther RW: Quantitative and qualitative assessment of left ventricular volume with ECG-gated multislice spiral CT: value of different image reconstruction algorithms in comparison to MRI Acta Radio/ 2003, 44(6):604-611.

15. Contreras S, Vazquez JM, Miguel AD, Morales M, Gil F, Lopez O, Arencibia A: Magnetic resonance angiography of the normal canine heart and associated blood vessels. Vet J 2008, 178(1):130-132

16. Henjes CR, Nolte I, Wefstaedt P: Multidetector-row computed tomography of thoracic aortic anomalies in dogs and cats: patent ductus arteriosus and vascular rings. BMC Vet Res 2011, 7:57.

17. Mai W, Weisse C, Sleeper MM: Cardiac magnetic resonance imaging in normal dogs and two dogs with heart base tumor. Vet Radiol Ultrasound 2010, 51(4):428-435.

18. McMurray JJ, Adamopoulos S, Anker SD, Auricchio A, Bohm M, Dickstein K, Falk V, Filippatos G, Fonseca C, Sanchez MA, Jaarsma T, Kober L, Lip GY, Maggion AP, Parkhomenko A, Pieske BM, Popescu BA, Ronnevik PK, Rutten FH, Schwitter J, Seferovic P, Stepinska J, Trindade PT, Voors AA, Zannad F, Zeiher A, Bax JJ, Baumgartner $\mathrm{H}$, Ceconi $\mathrm{C}$, Dean V, et al: ESC guidelines for the diagnosis and treatment of acute and chronic heart failure 2012: the task force for the diagnosis and treatment of acute and chronic heart failure 2012 of the European Society of cardiology. Developed in collaboration with the Heart Failure Association (HFA) of the ESC. Eur Heart J 2012, 14(8):803-869.

19. Kjaergaard J, Petersen CL, Kjaer A, Schaadt BK, Oh JK, Hassager C: Evaluation of right ventricular volume and function by $2 \mathrm{D}$ and $3 \mathrm{D}$ echocardiography compared to MRI. Eur J Echocardiogr 2006, 7(6):430-438.

20. Maffei E, Messalli G, Martini C, Nieman K, Catalano O, Rossi A, Seitun S, Guaricci Al, Tedeschi C, Mollet NR, Cademartiri F: Left and right ventricle assessment with Cardiac CT: validation study vs. Cardiac MR. Eur Radiol 2011, 22(5):1041-1049.

21. Markiewicz W, Sechtem U, Kirby R, Derugin N, Caputo GC, Higgins CB: Measurement of ventricular volumes in the dog by nuclear magnetic resonance imaging. J Am Coll Cardiol 1987, 10(1):170-177.

22. Shors SM, Fung CW, Francois CJ, Finn JP, Fieno DS: Accurate quantification of right ventricular mass at MR imaging by using cine true fast imaging with steady-state precession: study in dogs. Radiology 2004, 230(2):383-388.

23. Tamborini G, Marsan NA, Gripari P, Maffessanti F, Brusoni D, Muratori M, Caiani EG, Fiorentini C, Pepi M: Reference values for right ventricular volumes and ejection fraction with real-time three-dimensional echocardiography: evaluation in a large series of normal subjects. J Am SoC Echocardiogr 2010, 23(2):109-115.

24. Robbers-Visser D, Boersma E, Helbing WA: Normal biventricular function, volumes, and mass in children aged 8 to 17 years. J Magn Reson Imaging 2009, 29(3):552-559.

25. Hergan K, Schuster A, Fruhwald J, Mair M, Burger R, Topker M: Comparison of left and right ventricular volume measurement using the Simpson's method and the area length method. Eur J Radiol 2008, 65(2):270-278.

26. Cademartiri F, Luccichenti G, Gualerzi M, Brambilla L, Brambilla V, Coruzzi P: Intravenous contrast material administration in multislice computed tomography coronary angiography. Acta Biomed 2005, 76(2):86-94.

27. Smets $P$, Daminet $S$, Wess $G$ : Simpson's method of discs for measurement of echocardiographic end-diastolic and end-systolic left ventricular volumes: breed-specific reference ranges in boxer dogs. J Vet Intern Med 2014, 28(1):116-122.

28. Wess G, Mäurer J, Simak J, Hartmann K: Use of Simpson's method of disc to detect early echocardiographic changes in doberman pinschers with dilated cardiomyopathy. J Vet Intern Med 2010, 24(5):1069-1076.
29. Serres F, Chetboul V, Tissier R, Poujol L, Gouni V, Carlos Sampedrano C, Pouchelon $J$ : Comparison of 3 ultrasound methods for quantifying left ventricular systolic function: correlation with disease severity and prognostic value in dogs with mitral valve disease. J Vet Intern Med 2008, 22(3):566-577

30. Tidholm A, Westling AB, Höglund K, Ljungvall I, Häggström J: Comparisons of 3-, 2-dimensional, and M-mode echocardiographical methods for estimation of left chamber volumes in dogs with and without acquired heart disease. J Vet Intern Med 2010, 24(6):1414-1420.

31. Nakayama Y, Takaki M, Kohno K, Araki J, Suga H: Mechanoenergetics of the negative inotropism of isoflurane in the canine left ventricle. No O2 wasting effect. Anesthesiology 1997, 87(1):82-93.

32. Lan CC, Chang CY, Peng CK, Wu CP, Huang KL, Lee SC, Chang H: Effect of body positions on hemodynamics and gas exchange in anesthetized pigs shortly after pneumonectomy. Shock 2010, 34(5):482-487.

33. Siepe M, Ruegg DM, Giraud MN, Python J, Carrel T, Tevaearai HT: Effect of acute body positional changes on the haemodynamics of rats with and without myocardial infarction. Exp Physiol 2005, 90(4):627-634.

34. Nakao S, Come PC, Miller MJ, Momomura S, Sahagian P, Ransil BJ, Grossman W: Effects of supine and lateral positions on cardiac output and intracardiac pressures: an experimental study. Circulation 1986, 73(3):579-585.

35. Hoffmann R, Barletta G, von Bardeleben S, Vanoverschelde JL, Kasprzak J, Greis C, Becher $\mathrm{H}$ : Analysis of left ventricular volumes and function: a multicenter comparison of cardiac magnetic resonance imaging, cine ventriculography, and unenhanced and contrast-enhanced two-dimensional and three-dimensional echocardiography. J Am Soc Echocardiogr 2014, 27(3):292-301.

36. Laser KT, Bunge M, Hauffe P, Argueta JR, Kelter-Klopping A, Barth P, Sarikouch S, Burchert W, Kececioglu D, Korperich H: Left ventricular volumetry in healthy children and adolescents: comparison of two different realtime three-dimensional matrix transducers with cardiovascular magnetic resonance. Eur J Echocardiogr 2010, 11(2):138-148.

37. Simpson JM, Miller O: Three-dimensional echocardiography in congenital heart disease. Arch Cardiovasc Dis 2011, 104(1):45-56.

38. Perrin DP, Vasilyev NV, Marx GR, del Nido PJ: Temporal enhancement of 3D echocardiography by frame reordering. J Am Coll Cardiol Img 2012, 5(3):300-304.

39. Badano L, Lang RM, Zamorano JL: Textbook of Real-Time Three Dimensional Echocardiography. London: Springer London; 2010.

40. Laser KT, Horst JP, Barth P, Kelter-Klopping A, Haas NA, Burchert W, Kececioglu D, Korperich $\mathrm{H}$ : Knowledge-based reconstruction of right ventricular volumes using real-time three-dimensional echocardiographic as well as cardiac magnetic resonance images: comparison with a cardiac magnetic resonance standard. J Am Soc Echocardiogr 2014 27(10):1087-1097

41. Koch K, Oellig F, Oberholzer K, Bender P, Kunz P, Mildenberger P, Hake U, Kreitner KF, Thelen M: Assessment of right ventricular function by 16-detector-row CT: comparison with magnetic resonance imaging. Eur Radiol 2005, 15(2):312-318

42. Takx RA, Moscariello A, Schoepf UJ, Barraza JM Jr, Nance JW Jr, Bastarrika G, Das M, Meyer M, Wildberger JE, Schoenberg SO, Fink C, Henzler T: Quantification of left and right ventricular function and myocardial mass: comparison of low-radiation dose 2 nd generation dual-source CT and cardiac MRI. Eur J Radiol 2011, 81(4):e598-e604.

43. Sugeng L, Mor-Avi V, Weinert L, Niel J, Ebner C, Steringer-Mascherbauer R, Schmidt F, Galuschky C, Schummers G, Lang RM, Nesser HJ: Quantitative assessment of left ventricular size and function: side-by-side comparison of real-time three-dimensional echocardiography and computed tomography with magnetic resonance reference. Circulation 2006, 114(7):654-661.

44. Heinz-Peer G, Kopsa W: Richtlinien zur Kontrastmittelanwendung. Zeitschrift des Verbandes für Bildgebende Diagnostik Österreich 2005, 8(16):1-2.

45. Guo YK, Gao HL, Zhang XC, Wang QL, Yang ZG, Ma ES: Accuracy and reproducibility of assessing right ventricular function with 64-section multi-detector row CT: comparison with magnetic resonance imaging. Int J Cardiol 2010, 139(3):254-262.

46. Nesser HJ, Tkalec W, Patel AR, Masani ND, Niel J, Markt B, Pandian NG: Quantitation of right ventricular volumes and ejection fraction by threedimensional echocardiography in patients: comparison with magnetic resonance imaging and radionuclide ventriculography. Echocardiography 2006, 23(8):666-680. 
47. Dell'Italia LJ, Blackwell GG, Pearce DJ, Thorn B, Pohost GM: Assessment of ventricular volumes using cine magnetic resonance in the intact dog. A comparison of measurement methods. Invest Radiol 1994, 29(2):162-167.

48. Alfakih $\mathrm{K}$, Reid $\mathrm{S}$, Jones $\mathrm{T}$, Sivananthan M: Assessment of ventricular function and mass by cardiac magnetic resonance imaging. Eur Radiol 2004, 14(10):1813-1822.

49. Gilbert SH, McConnell FJ, Holden AV, Sivananthan MU, Dukes-McEwan J: The potential role of MRI in veterinary clinical cardiology. Vet J 2008, 183(2):124-134.

doi:10.1186/s12917-014-0242-3

Cite this article as: Sieslack et al.: Quantification of right ventricular

volume in dogs: a comparative study between three-dimensional echocardiography and computed tomography with the reference method magnetic resonance imaging. BMC Veterinary Research 2014 10:242.

\section{Submit your next manuscript to BioMed Central and take full advantage of:}

- Convenient online submission

- Thorough peer review

- No space constraints or color figure charges

- Immediate publication on acceptance

- Inclusion in PubMed, CAS, Scopus and Google Scholar

- Research which is freely available for redistribution 